\title{
La valorisation par $S / S$ à base de chaux et additifs de vases de dragage : résultats et perspectives
}

Francis Rey1, 2, Daniel Levacher1, Jean-Louis Quénec'h ${ }^{2}$

IUPRES A 6143 "Morphodynamique continentale et côtière', Université de Caen, 24, rue des Tilleuls, 14000 Caen.

${ }^{2}$ Ecole Supérieure d'Ingénieurs des Travaux de la Construction de Caen, 1 rue Pierre et Marie Curie, 14610 Epron.

\section{Résumé}

Devant la situation actuelle préoccupante du devenir des matériaux vasards contaminés, le traitement par Solidification/Stabilisation $(\mathrm{S} / \mathrm{S})$ est une solution pouvant permettre leur valorisation en génie civil.

Après une étude qui a permis d'identifier les mécanismes physico-chimiques clefs responsables des évolutions physico-mécaniques complexes de ce type de composites, une étude de lixiviation a été conduite afin d'étudier l'efficacité et le type d'immobilisation des métaux lourds au sein des matrices. Les résultats nous amènent à considérer différents potentiels d'immobilisation suivant les métaux considérés.

\begin{abstract}
Sludge materials pose a problem for harbour and coastal engineers in respect with the environment rules. The Stabilisation/ Solidification ( $\mathrm{S} / \mathrm{S}$ concept) constitutes one way to reuse them as civil construction materials. After determination of the fundamental mechanisms governing the complex physical-chemical evolutions of this composites, lixiviation tests were improved in order to study the efficiency and type of contaminants mobility. The results allow to consider different mobility potentials according to the studied metals.
\end{abstract}

\section{Introduction}

Les activités de dragage génèrent annuellement des volumes de matériaux vasards considérables (environ 50 millions de $\mathrm{m}^{3}$ en France; Alzieu 1999). Les gestions traditionnelles de ces matériaux semblent condamnées à plus ou moins court terme du fait de la raréfaction des sites de dépôts terrestres et suite à l'évolution de la réglementation pour les immersions en mer (GEODE 1994). En effet, une importante part de ces matériaux contiennent, selon les sites, des quantités non négligeables de contaminants si on ce réfaire aux seuils décrivant le bruit de fond géologique selon la grille GEODE. Devant cette situation la valorisation après traitement de $\mathrm{S} / \mathrm{S}$ est une solution alternative attrayante. Plusieurs études ainsi que des projets industriels, notamment au Japon (Kamon et al. 1990) et au Canada (Stegeman et al.1991) semblent confirmer l'intérêt de la technique. 
Dans ce cadre, des travaux visant à définir les propriétés mécaniques, physiques et environnementales des matériaux après une technique de solidification/stabilisation ont été menés. Parmi ceux-ci, le traitement des vases à la chaux plus additifs est une approche intéressante.

L'ajout de chaux vive à la vase brute et remaniée en sortie du dragage, confère rapidement aux vases une structure grumeleuse organo-minérale sitôt que la réaction fortement exothermique d'extinction a eu lieu. Les opérations de manipulation, stockage ou transport, sont alors simplifiées. A ce stade, l'obtention d'un matériau monolithique, susceptible de répondre aux exigences mécaniques et environnementales liées, par exemple, à une utilisation en construction routière, passe par l'ajout d'un liant et un compactage. Cette méthode permet de constituer un matériau monolithique, insoluble et le moins poreux possible.

\section{Caractéristiques et poliutions des vases utilisées}

Les vases utilisées proviennent du port de commerce du Havre (Seine-Maritime) et du port de plaisance de Dives-sur-Mer (Calvados). Les vases du Havre sont issues d'un dragage mécanique à godets alors que la vase de Dives provient d'un dragage hydraulique. Les principales caractéristiques physico-chimiques des prélèvements sont résumées dans le tableau 1.

Les deux vases sont semblables d'un point de vue granulométrique et minéralogique. Les phases majoritaires sont le quartz et les carbonates. Dans les deux cas également, les minéraux phyliteux sont peu présents. Ces caractéristiques sont une spécificité des sédiments côtiers de la baie de Seine. La solution interstitielle contient des sels dissous provenant de la nature marine des matériaux. La valeur du Carbone Organique Total (COT) suffit à donner un caractère nettement organique au produit en constituant un gel organométallique noir-brun caractéristique des matériaux vasards et de leurs propriétés particulières de consolidation et de viscosité. La matière organique est principalement constituée de Substances Humifiées ( $\mathrm{SH}$ ) dont les constituants sont l'humine (insoluble et fortement associée à la phase minérale), les acides humiques (solubles en milieu alcalin) et les acides fulviques en solution. Ces composés, qui sont des polymères complexes de structures non définies à noyaux aromatiques, ont de fortes propriétés de complexation avec les éléments et particules chargées (Busnot 1991). Ils constituent de ce fait les principaux agents de concentration et de rétention des contaminants au sein du matériau vasard après floculation/coagulation et décantation des agrégats minéralo-organiques.

La teneur totale des contaminants métalliques est obtenue après minéralisation totale de l'échantillon conformément à la norme NF X 31-151. Le dosage est ensuite réalisé après reprise des cendres par spectrométrie d'absorption atomique au four. Les résultats sont présentés dans le tableau 1.

D'après la recommandation GEODE (1994), la vase de Dives, pour ces métaux, est faiblement nuisible pour l'environnement marin dans la mesure où la contamination est comprise entre le Bruit de Fond "géologique" (BdF) et le niveau 1 de cette grille. Pour la vase du Havre, la situation est différente. Les teneurs en $\mathrm{Cd}, \mathrm{Cr}, \mathrm{Cu}$ 
et $P b$ sont comparativement bien supérieures à la vase de Dives et sont supérieures au niveau 1. Dans ce cas, l'immersion comporte un risque potentiel necessitant, d'après la recommandation GEODE, une étude complémentaire, d'autant plus que le cadmium $(C d)$ qui est particulièrement toxique, semble présent à une teneur supérieure à 3 fois le BdF. Pour ce site, trouver une solution alternative à l'immersion apparaît donc important. Le sédiment étudié est, de plus, plutôt représentatif de la moyenne des vases draguées en appartenant à une campagne de dragage ordinaire et ne constitue en rien un échantillon particulièrement pollué.

\begin{tabular}{|c|c|c|c|c|c|c|c|c|}
\hline \multicolumn{3}{|c|}{ Caractéristiques physico-chimiques des vases } & \multicolumn{6}{|c|}{ Teneur en contaminants métalliques ${ }^{4}$} \\
\hline & Dives & $\begin{array}{c}\text { Le } \\
\text { Havre }\end{array}$ & $(\mathrm{mg} / \mathrm{kg})$ & Dives & $\begin{array}{c}\text { Le } \\
\text { Havre }\end{array}$ & $\mathrm{BdF}^{5}$ & Niv. $1^{5}$ & Niv. $2^{5}$ \\
\hline Teneur en eau : w $(\%)^{1}$ & $170-182$ & $128-134$ & $\mathrm{Cd}$ & 0,72 & 1,64 & 0,5 & 1,2 & 2,4 \\
\hline Densité de la phase solide : $\mathrm{d}^{1}$ & 2,65 & 2,63 & $\mathrm{Cr}$ & 75 & 109 & 45 & 90 & 180 \\
\hline Indice de plasticité $: I_{p}(\%)^{1}$ & 5 & 55 & $\mathrm{Cu}$ & 35 & 56 & 35 & 45 & 90 \\
\hline Argile $(<\grave{a} 2 \mu \mathrm{m})(\%)^{2}$ & 23 & 31 & $\mathrm{Ni}$ & 23 & 23 & 20 & 37 & 74 \\
\hline Silt $(2$ à $64 \mu \mathrm{m})(\%)^{2}$ & 73 & 67 & $\mathrm{~Pb}$ & 57 & 102 & 47 & 100 & 200 \\
\hline Sable $(>$ à $64 \mu \mathrm{m})(\%)^{2}$ & 4 & 2 & $\mathrm{Zn}$ & 195 & 209 & 115 & 276 & 552 \\
\hline Indice de floculation : If $(\%)^{3}$ & 60 & 46 & \multirow{8}{*}{\multicolumn{6}{|c|}{ 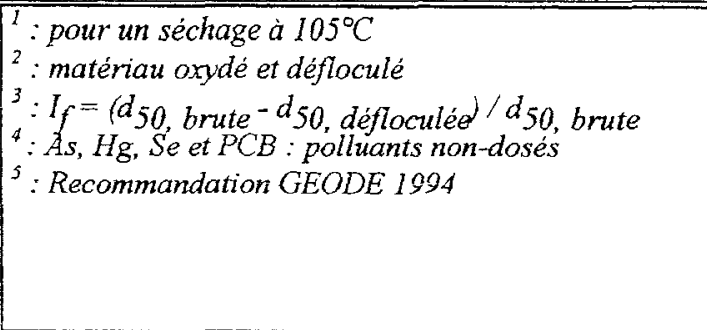 }} \\
\hline Quartz $(\%)$ & 40 & 43 & & & & & & \\
\hline Carbonates $(\%)$ & 42 & 38 & & & & & & \\
\hline Feldspaths et micas (\%) & 5,5 & 3 & & & & & & \\
\hline Argiles (\%) & 3 & 3 & & & & & & \\
\hline Chlorures $(\%)$ & 3,08 & 2,40 & & & & & & \\
\hline Sulfates $(\%)$ & 0,41 & 0,17 & & & & & & \\
\hline $\operatorname{CoT}(\%)$ & 2,88 & 3,23 & & & & & & \\
\hline
\end{tabular}

Tab. 1 : Caractéristiques physico-chimiques des vases et teneurs en contaminants.

Tab. I : Physico-chemical sludges caracteristics and contaminants contents.

\section{Paramètres du procédé de $\mathrm{S} / \mathrm{S}$ et modèle physique déterminé}

Pour cette étude, deux types d'ajouts ont été employés : un ciment CPJ CEM II A / $32,5 \mathrm{R}$ courant et des Cendres Volantes (CV) de houille de centrale thermique.

Avec ces deux types d'ajouts, employés séparément, il a été possible d'apprécier l'effet distinct des deux familles de liants courants appliqués à la solidification/stabilisation. Le CPJ représentera la famille des liants hydrauliques anhydres alors que les CV seront les représentantes des liants pouzzolaniques catalysés par les solutions de chaux.

Le type et la classe du ciment, à dosages modérés, ayant peu d'influence sur la résistance des composites vase-ciment (Boutouil 1998), le choix s'est donc porté sur le plus économique. Les cendres volantes retenues rentrent dans la composition moyenne des produits de ce type.

Le malaxage des constituants est réalisé en deux fois afin de bénéficier au maximum des propriétés d'absorption et d'évaporation d'eau de la chaux ainsi que de la floculation organo-calcique qui transforme radicalement les vases et minimise les interférences SH-liant. Le mélange de la vase avec la chaux précède donc (1/2 journée) celui réalisé avec l'ajout. Par ailleurs, pour obtenir la teneur en eau du 
mélange, le phénomène d'absorption et d'évaporation d'eau a été modélisé (corrélation : $r=0,99$ ):

$$
w_{\text {vase- chaux }}=\frac{w-x(1+w)\left(\alpha_{c h x}+\beta_{c h x}\right)}{1+x(1+w)\left(1+\alpha_{c h x}\right)}
$$

avec : Wvase-chaux : teneur en eau du mélange vase chaux après maturation ;

$x$ : dosage en chaux (masse de chaux / masse de vase brute) ;

$w$ : teneur en eau de la vase brute ;

$\alpha_{c h x}:$ rapport massique d'hydratation de la chaux ;

$\beta_{c h x}$ : rapport massique d'évaporation de la chaux.

Le dosage en chaux peut alors être déterminé suivant la teneur en eau désirée du mélange, celle-ci influençant la consistance pour la mise en œuvre. Le dosage en ajout $(y)$ est exprimé par rapport à la masse de mélange vase-chaux après maturation. Les dosages employés sont présentés dans le tableau 2.

\begin{tabular}{|c|c|c|c|c|c|c|c|c|}
\hline \multirow[t]{2}{*}{ Vase } & \multirow[t]{2}{*}{$\begin{array}{l}\text { Chaux } \\
x(\%)\end{array}$} & \multirow[t]{2}{*}{$\begin{array}{l}\text { Ajout } \\
y(\%)\end{array}$} & \multirow[t]{2}{*}{$\begin{array}{l}\text { FCV } \\
\text { Ciment }\end{array}$} & \multirow[t]{2}{*}{$\begin{array}{l}\mathrm{FCV}^{1} \\
\mathrm{CV}\end{array}$} & \multicolumn{2}{|c|}{ Légendes des figures } & \multicolumn{2}{|c|}{$\begin{array}{c}\text { Porosité totale initiale } \\
(\%)^{2}\end{array}$} \\
\hline & & & & & Ajout Ciment & Ajout CV & Ajout Ciment & Ajout CV \\
\hline Dives & $49-52$ & 7 & 1,09 & 1,15 & D-52-7-C & D-52-7-CV & 43,6 & 42,1 \\
\hline & $49-52$ & 17 & 1,15 & 1,28 & D-52-17-C & D-52-17-CV & 43,5 & 46,6 \\
\hline , & $49-52$ & 29 & 1,28 & 1,37 & D-52-29-C & D-52-29-CV & 42,5 & 45,6 \\
\hline 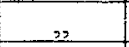 & 42 & 18 & 1,12 & 1,18 & $\mathrm{D}-42-18-\mathrm{C}$ & D-42-18-CV & 44,5 & 47,2 \\
\hline 23 & 36 & 27 & 1,14 & 1,24 & D-36-27-C & D-36-27-CV & 50,3 & 47,0 \\
\hline Le Havre & $38-40$ & 7 & 1,07 & 1,10 & $\mathrm{H}-39-7-\mathrm{C}$ & $\mathrm{H}-39-7-\mathrm{CV}$ & 47,1 & 43,5 \\
\hline 3 & $38-40$ & 17 & 1,12 & 1,19 & $\mathrm{H}-39-17-\mathrm{C}$ & $\mathrm{H}-39-17-\mathrm{CV}$ & 41,5 & 45,0 \\
\hline 23 & $38-40$ & 29 & 1,21 & 1,33 & $\mathrm{H}-39-29-\mathrm{C}$ & $\mathrm{H}-39-29-\mathrm{CV}$ & 38,2 & 46,9 \\
\hline 3 & 33 & 18 & 1,13 & 1,20 & $\mathrm{H}-33-18-\mathrm{C}$ & $\mathrm{H}-33-18-\mathrm{CV}$ & 48,5 & 47,1 \\
\hline$\because$ & 27 & 27 & 1,17 & 1,26 & $\mathrm{H}-27-27-\mathrm{C}$ & $\mathrm{H}-27-27-\mathrm{CV}$ & 45,5 & 50,8 \\
\hline
\end{tabular}

' $:$ Facteur de Changement de Volume $=$ Volume composite $/$ Volume vase brute

2: Calculée à partir de la densité des phases solides (mesurée avec un pycnomètre azote multivolume en gaz réel sur le matériau désagrégé, Rey 1999) et la masse volumique apparente des éprouvettes.

Tab. 2 : Dosages et FCV du procédé de $\mathrm{S} / \mathrm{S}$; porosité totale des composites.

Tab. 2 : Mixtures and VCF of $S / S$ process; total porosity of composites.

Immédiatement après le malaxage avec l'ajout, des éprouvettes sont réalisées par compactage quasi statique dans des moules cylindriques suivant un mode opératoire analogue à celui de la norme NF P 98-230-2 sur les sols traités. Les éprouvettes, de $38,6( \pm 0,1) \mathrm{mm}$ de diamètre sur $77( \pm 1) \mathrm{mm}$ de hauteur ont, après extrusion, une masse volumique équivalente $( \pm 1 \%)$ à celle obtenue préalablement après un essai Proctor modifié (NF $\mathrm{P}$ 94-093). Ainsi les caractéristiques mécaniques et physico-chimiques des éprouvettes sont déterminées pour une consistance correspondant à une énergie de compactage constante. Notons également que les dosages minimaux employés, et particulièrement celui en chaux, sont une conséquence directe de cette méthodologie de confection et notamment de la faisabilité de réalisation de l'essai Proctor. Les éprouvettes sont ensuite conservées en moules hermétiques à $20( \pm 2)^{\circ} \mathrm{C}$.

Les valeurs du Facteur de Changement de Volume (FCV) montrent systématiquement, qu'à dosages identiques, les composites avec ajout de CV sont 
moins compressibles que ceux obtenus avec un ajout de type ciment. Ceci est la conséquence de la densité absolue plus élevée du ciment et de son hydratation avant compactage, alors que les CV sont à considérer, à très court terme, comme une poudre inerte.

\section{Problématique du traitement et concepts physico-mécaniques}

\subsection{Interférences des SH sur les cinétiques de formation des hydrates}

Lors du traitement des vases avec les liants hydrauliques, les Substances Humiques (SH) interfèrent fortement sur les processus d'hydratation ou de formation des phases cimentaires. Les composés organiques agissent probablement comme des agents retardateurs de prise : adsorption sur les surfaces anhydres, complexation avec les ions en solution (par analogie avec l'effet des adjuvants : Jolicoeur et al. 1998). Ils agissent également ici par immobilisation physique des ions dans le gel organique. Ces interférences sont très importantes et font considérablement chuter la résistance des matériaux à court et moyen terme (Kujala et al. 1996).

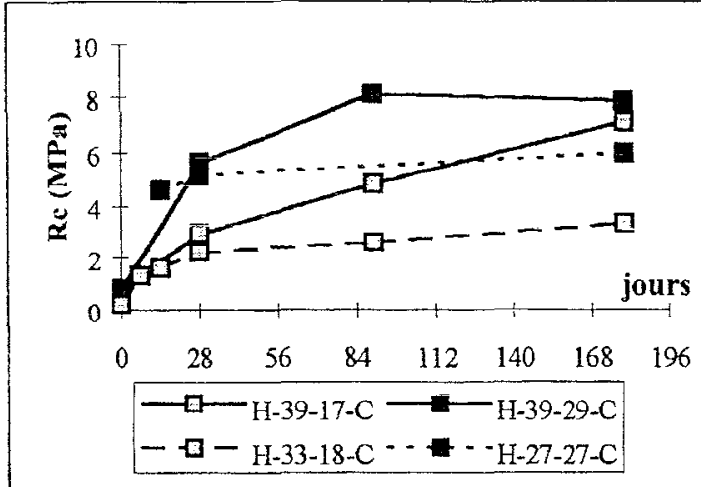

Fig. 1.a : Composites avec ajout de ciment

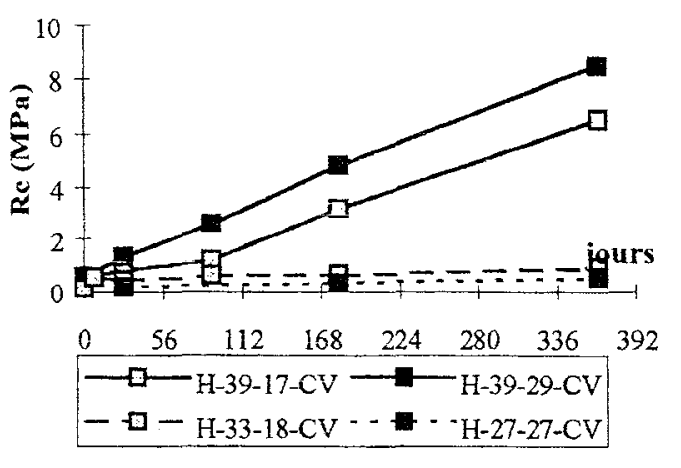

Fig. 1.b : Composites avec ajout de CV

Fig. 1 : Evolution des résistances en compression; influence du dosage en chaux.

Fig. $l$ : Evolution of simple compression resistance; lime mixture influence.

L'action du dosage préliminaire en chaux joue un rôle déterminant dans la cinétique de ce processus retardateur (voir figure 1).

Chimiquement la forte concentration en $\mathrm{Ca}^{2+}$, conduit à saturer la capacité d'échange cationique des SH. Les futures interférences sur les liants sont ainsi réduites. La chaux est également susceptible de développer une réaction pouzzolanique avec les minéraux naturels résultant de la mise en solution de fines particules minérales peu ou non cristallisées suite à l'attaque de la solution calcobasique (Le Roux 1969). Ceci conduit normalement, à plus ou moins long terme, à la formation, entre autres, de silicates de calcium hydratés (CSH) stables semblables à ceux formés par les ciments et aboutit donc à une solidification. Cependant à court et moyen terme le caractère nettement organique des vases inhibe totalement ce type de réaction (Rey et al. 1998). Outre les effets physicochimiques inhibiteurs précédemment cités dans les références, il faut ajouter le rôle de membrane protectrice que constitue l'humine autour des minéraux. 
Physiquement, le dosage en chaux est la cause de la floculation immédiate d'agrégats structuraux minéralo-organiques par complexation multiples $\mathrm{SH}-\mathrm{Ca}-\mathrm{SH}$ (Rey 1999). Ceux-ci viennent initialement jouer le rôle de "gramulats visco-élastoplastiques" dans les composites alors que les flocons formés sont d'autant plus denses que le milieu est asséché par la formation de portlandite $(\mathrm{CH})$.

\subsection{Minéralisation / transformation graduelle des SH}

La solution calcobasique des matrices conduit à l'oxydation des $\mathrm{SH}$ ce qui entraîne leur solubilisation/ minéralisation progressive. Cette transformation a de nombreuses conséquences prévisibles et entre autres :

- une libération des éléments complexés $(\mathrm{Ca}, \mathrm{Al}, \mathrm{Fe} \ldots)$ ainsi que des polluants ;

- une disponibilité des anions constitutifs des matières organiques $\left(\mathrm{CO}_{3}{ }^{2-} ; \mathrm{SO}_{4}{ }^{2-} \ldots\right)$; - une déstructuration des agrégats minéralo-organiques formés initialement.

Physiquement, la minéralisation/transformation des SH se traduit par des pertes plus ou moins importantes de matières sèches (voir figure 2).

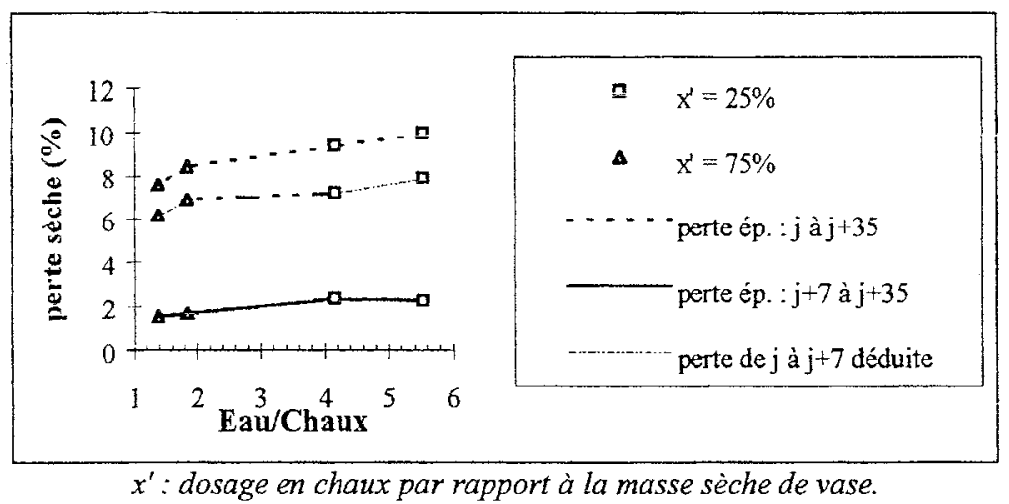

Fig. 2 : Perte sèche en fonction du rapport Eau/Chaux.

Fig. 2 : Dry matters loss versus Water/Lime ratio.

Ainsi, peu à peu, l'activité chimique pour la formation des CSH peut se développer dans le cas où celle-ci était initialement entravée (dosages faibles). Ceci conduit alors à une solidification efficace du matériau : insolubilité dans l'eau et résistance de plusieurs $\mathrm{MPa}$. Ce matériau devient alors potentiellement utilisable en génie civil (construction routière).

\subsection{Interférences structurelles des SH}

La formation de la matrice minérale se fait malgré tout en présence de forte concentration en polymères humiques. Les composés organiques, restent donc piégés dans les amas de précipitation et altèrent structurellement les produits d'hydratation. La cinétique de réaction du liant est alors en interaction avec celle de la minéralisation/transformation des $\mathrm{SH}$, ce qui a d'importantes conséquences.

La disparition des polymères humiques conduit à la disparition de l'armature polymérisée des structures minéralo-organiques et ainsi, peu à peu, à une déstructuration des agrégats organo-calciques. Dans la mesure où une structure 
coalescente minérale rigide les réunissait (dans le cas d'un liant à développement rapide des $\mathrm{CSH}$ ) des faiblesses internes apparaissent. Ceci est probablement la cause microstructurelle des chutes des caractéristiques mécaniques à long terme (voir figure 3). Dans le cas d'un développement lent des $\mathrm{CSH}$, ce mécanisme semble ne plus exister. En effet, aux membranes organiques des minéraux et aux liens organo-calciques se substituent probablement graduellement des liens CSH au cœur des structures floculées. La pérennité des caractéristiques mécaniques est alors assurée (voir figure 3 ).

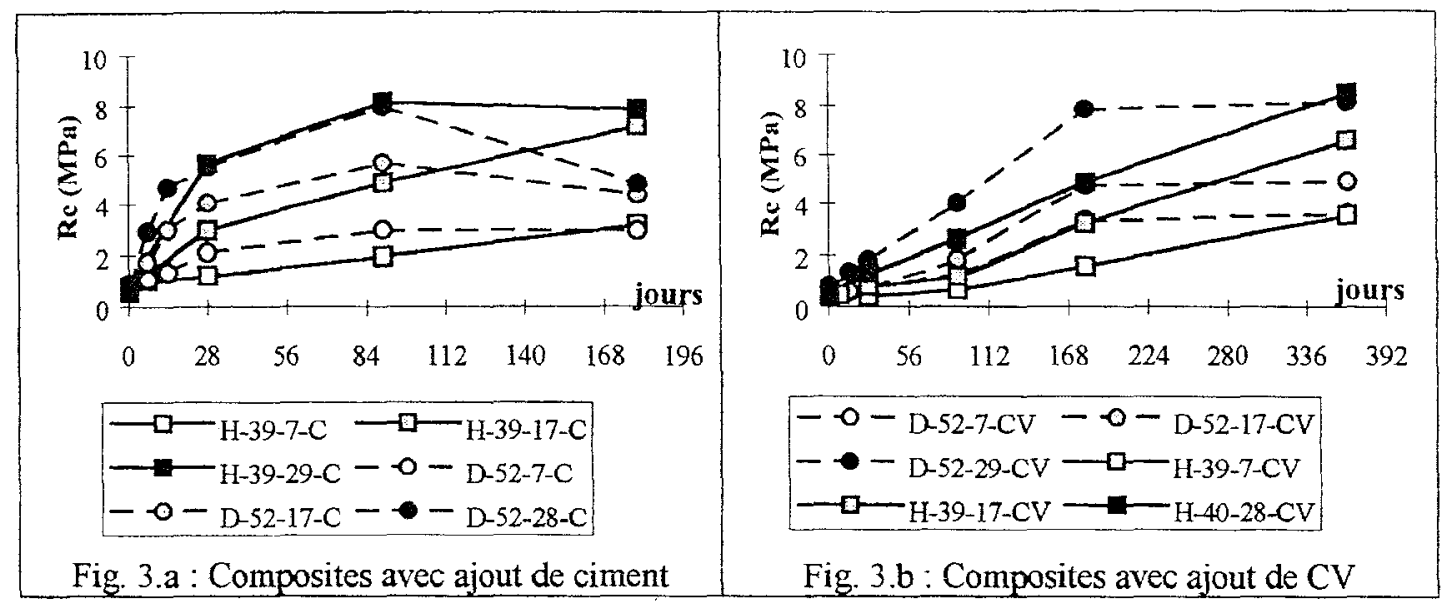

Fig. 3 : Evolution des résistances en compression simple; influence du dosage en liant et de la cinétique de réaction.

Fig. 3 : Evolution of simple resistance compression; binder mixture and cinetique reaction influence.

Dans cette hypothèse, l'utilisation de liants et d'additifs susceptibles d'aboutir à un développement rapide des $\mathrm{CSH}$ semble donc à proscrire. L'emploi d'un liant pouzzolanique est par conséquent intéressant si l'on ne recherche pas une rigidification rapide. Une combinaison des deux types de liant semble néanmoins la plus intéressante dans le cas courant.

\section{Spécificité physico-chimiques des matrices et étude environnementale}

\subsection{Développement des phases chloroaluminates}

L'étude physico-chimique révèle qu'il y a une précipitation initiale de monochloroaluminates de calcium hydratés $(\mathrm{CAC} / \mathrm{H})$ et que celle-ci se poursuit probablement très longtemps dans le temps au fur et à mesure que la minéralisation/transformation des $\mathrm{SH}$ s'opère. En effet, suivant les concentrations interstitielles respectives de $\mathrm{CaCl}_{2}, \mathrm{CaSO}_{4}, \mathrm{H}_{2} \mathrm{CO}_{3}$, et $\mathrm{Al}_{2} \mathrm{O}_{3}$, la libération de ces divers éléments rend de nouveau théoriquement possible la formation des chloro(sulfo, calco)aluminates de calcium hydratés. La formation de monochloroaluminates est longtemps la plus probable. Les sulfoaluminates n'ont pas été détectés par DRX et la présence de chlorures dans la solution interstitielle rend leur présence improbable (Capmas 1994). A long terme, après épuisement des 
chlorures et minéralisation des $\mathrm{SH}$, leur formation devient théoriquement de nouveau possible. Ces formations tardives sont nuisibles dans le cas où leur mode porosimétrique intraflocons serait supérieur à ceux de la matrice déjà formée. Ceci semble être le cas pour les $\mathrm{CAClH}$ dans les matrices $\mathrm{CH}$ (voir figure 4), ce qui expliquerait les faibles gonflements observés à très long terme (Rey 1999).

\subsection{Porosité des matrices}

En ce qui concerne la porosité intrinsèque des matrices, l'étude porosimétrique au mercure (voir figure 4) a révélé que celle-ci était trimodale et correspondait probablement à l'emboîtement des systèmes minéraux des $\mathrm{CSH}$, des complexes minéralo-organiques calciques (complexes $\mathrm{CH}$ ) et des $\mathrm{CAC} H$. Cette porosité possède un volume poreux maximum pour le système des complexes $\mathrm{CH}$, ce qui est en rapport avec les dosages importants en chaux. Le système CSH est peu présent ce qui vient des faibles dosages en ajouts. Une macroporosité capillaire est présente dans le cas où la matrice ne s'effondrerait pas sur elle-même suite aux pressions de succion qu'entraîne l'auto-dessiccation et le séchage de l'échantillon pour l'essãai. Dans le cas de l'ajout $\mathrm{CV}$, ce mode porosimétrique semble provenir principalement de la désagrégation des sphénles de verre des CV dans la matrice sous l'action de la solution de chaux. Cette macroporosité capillaire est, par conséquent, bien plus faible dans le cas de la matrice chaux-ciment.

Pour les composites vase-chaux-CV, et par rapport au composite avec ajout de ciment, il a par ailleurs été constaté un léger décalage et un élargissement des modes porosimétriques attribués aux structures minéralo-organiques calciques (complexes $\mathrm{CH}$ ) et $\mathrm{CACl}$. Ces variations, avec la porosité totale un peu plus élevée des composites $\mathrm{CV}$, sont probablement liées à l'auto-dessiccation initialement moins importante du milieu au moment du compactage. Les valeurs de porosité totale $(\mathrm{n})$, pour un même dosage en chaux, se classent normalement dans l'ordre des dosages en ajout mais le dosage en chaux reste déterminant pour cette valeur qui est contrôlée par la baisse de la teneur en eau du matériau (tableau 2).

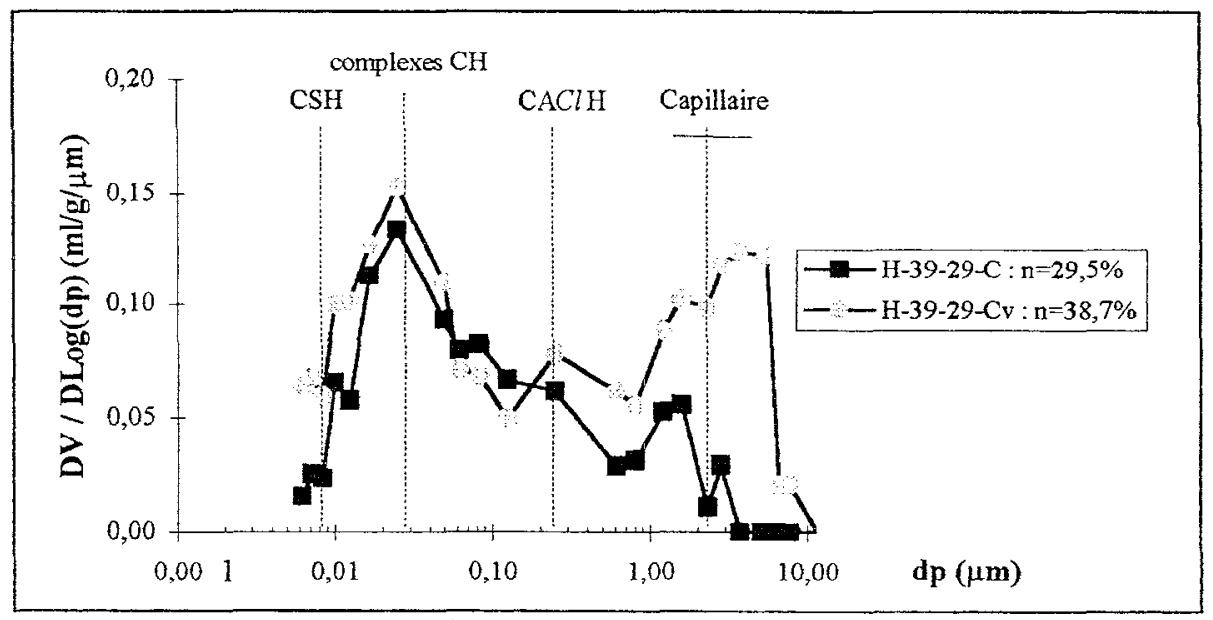

Fig. 4 : Modes porosimétriques des matrices et identifications avancées.

Fig. 4 : Porosity modes of matrix and advanced identification. 


\subsection{Mode d'immobilisation et lixiviation des métaux}

L'importance de la porosité des matériaux va avoir une influence sur la lixiviation des polluants solubles par l'intermédiaire de la perméabilité. Dans le cas où la fixation chimique minérale des polluants serait peu efficace, comme cela est probablement le cas à court et moyen terme (métaux restés complexés avec les $\mathrm{SH}$ ), les composites vase-chaux-CV se révéleront moins efficaces que ceux avec ajout de ciment du fait de leur porosité plus importante avec un mode capillaire également plus important.

Des essais de lixiviation font apparaitre (voir figure 5) que pour $\mathrm{Cu}$ et $\mathrm{Ni}$, métaux sensibles à la solubilisation des complexes en milieu basique (Calmano et al. 1983), cette tendance semble vérifiée. En effet, à dosages semblables, la lixiviation sur éprouvette monolithique des composites $\mathrm{CV}$ est toujours légèrement plus importante que pour le ciment. De plus, nous constatons que l'immobilisation physique de ces métaux est peu efficace dans la mesure où la lixiviation sur monolithe est assez proche de la lixiviation sur agrégats.

Le plomb et le zinc sont par contre mieux immobilisés physiquement, comme la différence des deux modes de lixiviation le laisse penser (voir figure 5). Ces métaux semblent de plus insensibles aux différences de porosité des matrices. Ces deux éléments sont, a priori, immobilisés par les phases aluminates $(\mathrm{CAC} / \mathrm{H})$, qui sont particulièrement aptes aux substitutions avec $\mathrm{Pb}$ et $\mathrm{Zn}$ (Capmas et al. 1994). Néanmoins, on constate que la fixation chimique avec l'ajout ciment est meilleure. Enfin, il a été établi que la lixiviation des chlorures était bien corrélée avec la porosité totale des composites (voir figure 5), ce qui suggère que la lixiviation des espèces solubles est effectivement contrôlée par celle-ci alors que l'importance de la porosité capillaire ("macroporosité") a peu d'influence sur ce phénomène.

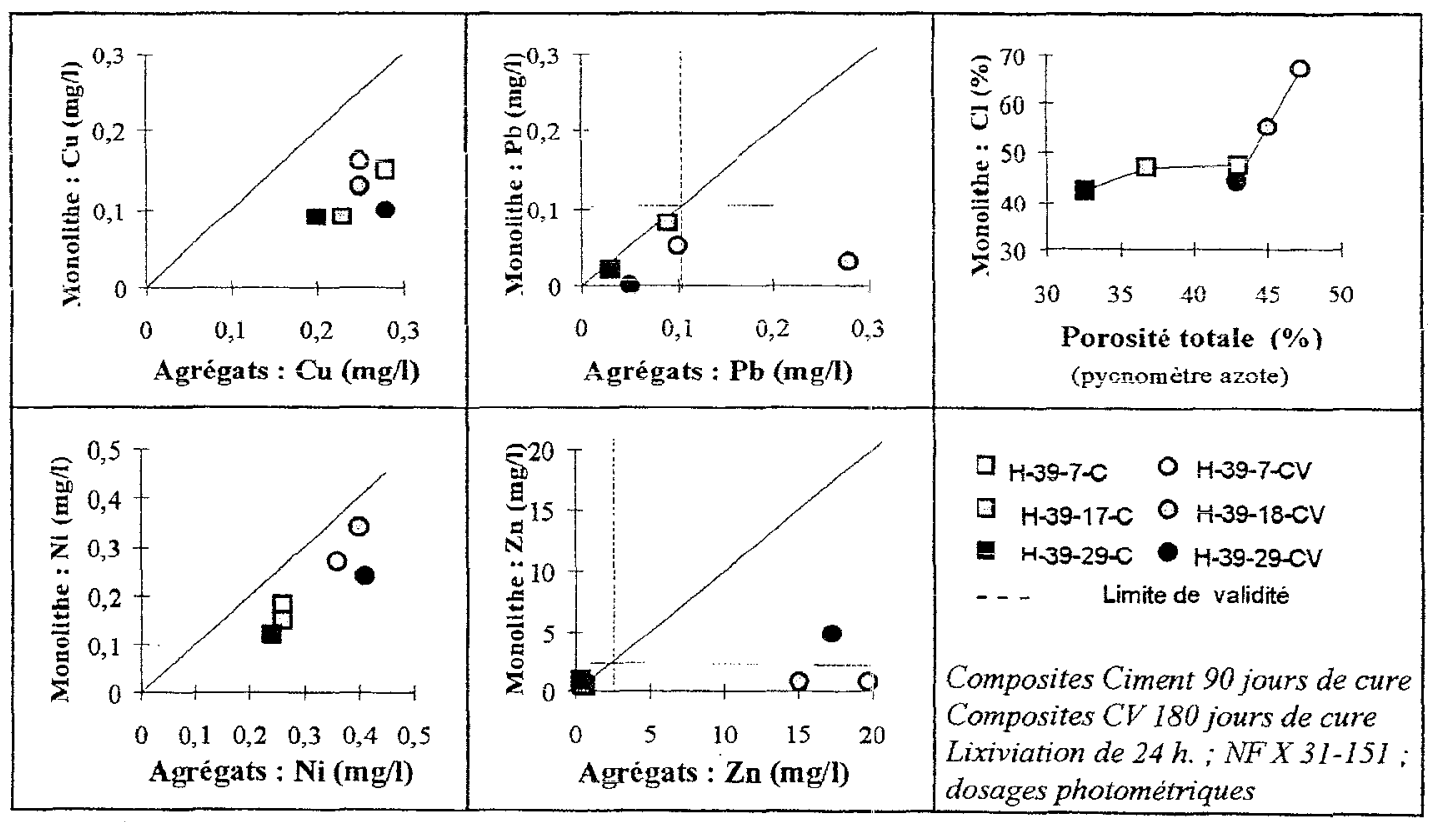

Fig. 5 : Résultats des lixiviations et corrélation porosité - chlorures lixiviés.

Fig. 5 : Lixiviations results and correlation porosity - lixivied chlorides 


\section{Conclusions et perspectives}

Nous conclurons en précisant que le traitement des vases de dragage portuaires par solidification/stabilisation à base de chaux pour constituer des composites cimentiers ou/et pouzzolaniques susceptibles de répondre à une utilisation dans le domaine du génie civil se heurte à certains facteurs comportementaux comme la pérennité des résistances et la lixiviation du $\mathrm{Cu}$ et du $\mathrm{Ni}$ ainsi qu'économiques liés à l'utilisation de dosages importants. Cependant, en tenant compte de la synthèse des conclusions phénoménologiques de cette étude, certaines possibilités s'ouvrent pour améliorer ces critères :

- d'une part l'étude d'un prétraitement des vases pour minimiser l'influence des matières organiques sur le procédé de $S / S$. Un processus d'oxydation accéléré peut être envisagé ;

- d'autre part l'utilisation d'un liant spécifique adapté à la triple problématiques forte teneur en eau - matériau organique - contaminants métalliques. Un liant pouzzolanique sulfo-alumino-calcique parait être une solution à la lumière de l'étude réalisée.

\section{Références}

Aizieu C., 1999. Dragage et environnement marin - Etat des connaissances. Ed. IFREMER, 224p.

Boutouil M., 1998. Traitement des vases de dragage par solidification/ stabilisation à base de ciment et additifs. Thèse de Doctorat, Le Havre, 258p.

Busnot I., 1991. Les substances humiques : Complexants naturels. Thèse de Doctorat, Caen, $202 \mathrm{p}$.

Calmano W., Förstner U., Kersten M., Kraus D., 1983. Behavior of dredged mud after stabilisation with different additives. Sci. Total Environ., pp 737-747.

Capmas A. et Sorrentino F., 1994. Etude des mécanismes de solidification-stabilisation des déchets solides par liants hydrauliques - cas des Refiom. Environnement et Technique, $\mathrm{n}^{\circ} 136$, pp. 50-53.

GEODE, 1994. Dragage dans les ports français. Rapport de synthèse SOGREAH, $113 \mathrm{p}$.

Jolicoeur C. et Simard M.-A., 1998. Chemical admixture-cement interactions : phenomenology and physico-chemical concepts. Cement and Con. Comp., $\mathrm{n}^{\circ} 20, \mathrm{pp} .87-101$.

Kamon M. et Nontananandh S., 1990. Contribution of stainless steel slag to the development of strength for seabed hedoro. Japanese Society of Soil Mechanics and Foundation Eng., Vol. 30, $n^{\circ}$ 4, pp. 63-72.

Kujala K. et Mäkikyrö M., 1996. Effect of humus on the binding reaction in stabilized soils. Grouting and Deep Mixing, Ed. Yonekura Terashi et Shibazaki, Rotterdam, pp. 415-420.

Le Roux A., 1969. Contribution à l'étude du traitement à la chaux des matériaux argileux. Thèse de Doctorat, Paris, Orsay, 105 p.

Rey F., Levacher D., Bennabi A., Quenec'h J-L., 1998. Etude du comportement mécanique et des conditions de mise en oeuvre de composites vase-chaux. 16èmes Rencontres universitaires de génie civil, Reims, pp 122-129.

Rey F., 1999. Etude physico-mécanique et environnementale du composite vase-chaux et additifs. Thèse de Doctorat, Caen, $271 \mathrm{p}$.

Stegemann J.A. et Cote P.L., 1991. Programme conjoint d'étude de méthodes d'essais pour l'évaluation des déchets solidifiés. Environ. Canada SPE3/HA/8, 13lp. 